ISSN 1014-4874

DOI : http://dx.doi.org/10.4314/rj.v27i1.3

\title{
A Conceptual Model for Remote Data Acquisition Using SMSLib Software. Case study: Kivu Lake Water Level
}

1. Cesar Niyomugabo, National University of Rwanda, Department of Electrical and Electronics Engineering

2. Célestin Twizere, National University of Rwanda, Department of Electrical and Electronics Engineering - Corresponding author Email: ctwizere@nur.ac.rw

\begin{abstract}
This paper presents a design of a remote lake-water-level measurement data acquisition system via UMTS network. The system accomplishes the function of data processing and transmitting by the use of SMSlib software and a java application developed. A tree layer system is designed to achieve this work: a mobile station layer, UMTS network layer and a sensing part layer. The system is more flexible than using server based network.

From a mobile phone, via a UMTS network layer an SMs is sent to a computer $(P C)$ on which a pressure sensor is immersed in water and connected on other terminal. Then the computer replies to the request using an SMs after processing information about the level of water received from the sensor.

As a low cost, good and high performance and easy maintainable data acquisition system based on SMSlib software, this type of data acquisition system can be applied not only in the fields of hydrological processes, but also in fields like civil engineering and in some more industrial requirements solutions.
\end{abstract}

Keywords- Remote Data Acquisition, UMTS Network, Water level, Pressure Sensor.

\section{Introduction}

The main problem that rose the thought about this conception was the daily need of the Kivu lake level measurement, this in order to optimize the use of resource while keeping natural environmental resource safety during the production of electricity by SINELAC/CEPGL. The major focus of this paper was to design a remote lake-water-level measurement data acquisition system that is sensor, SMSlib open source and UMTS network based.

The system used by SINELAC today is a classic one where people, each morning go to get the data about the level at the lake. With the new system the man power is supported because data are captured remotely. This will be a fully new system introduced.

A top-down design strategy approach is taken here. From a mobile phone, via a UMTS network (Naral Dalal, 2001) an SMs is sent to a computer (PC) 
on which a pressure sensor immersed in water and connected on other terminal. The computer responds to the request by sending an SMs after processing information about the level of water received from the sensor. To support this service, a tree layer system is designed: Mobile station layer, UMTS network layer and Sensing part layer. This must be a fixed application with an analog input signal with voltage range between 0.5 and 2.5 Volts (Global Water, 2009).

\section{Designing the System Topology}

To get started with design of the system, first we need to know the behavior of the Kivu lake level. The variations in the global hydrological cycle occur at many time and space scales, these variations may result from local factors or climate change. However, spatial climatically-induced patterns can be identified when most lakes in a region change in a similar way. We want to make measurement of the Kivu lake level because out of this lake comes Rusizi river on which power plants that that require Kivu level monitoring are placed.

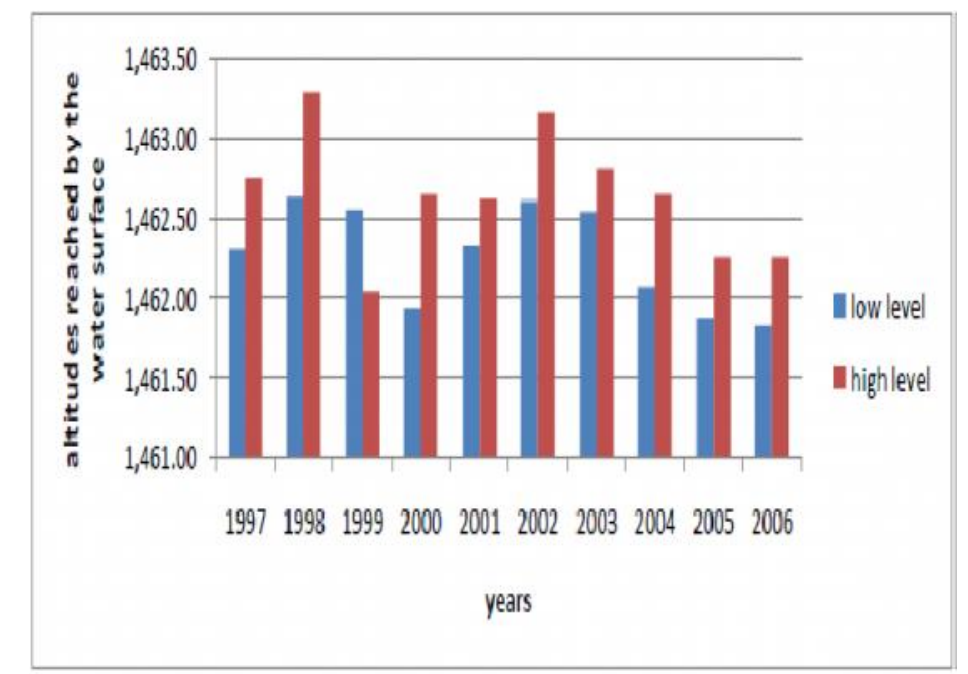

Figure 1: Kivu lake levels reached for each year since 1997

On the figure above, we can see that the highest level measured is 1463.283 meter measured in 1998 and the lowest level faced is 1462.823 measured in 2006. Figure 2 shows the difference between high and low level per year since 1997. 


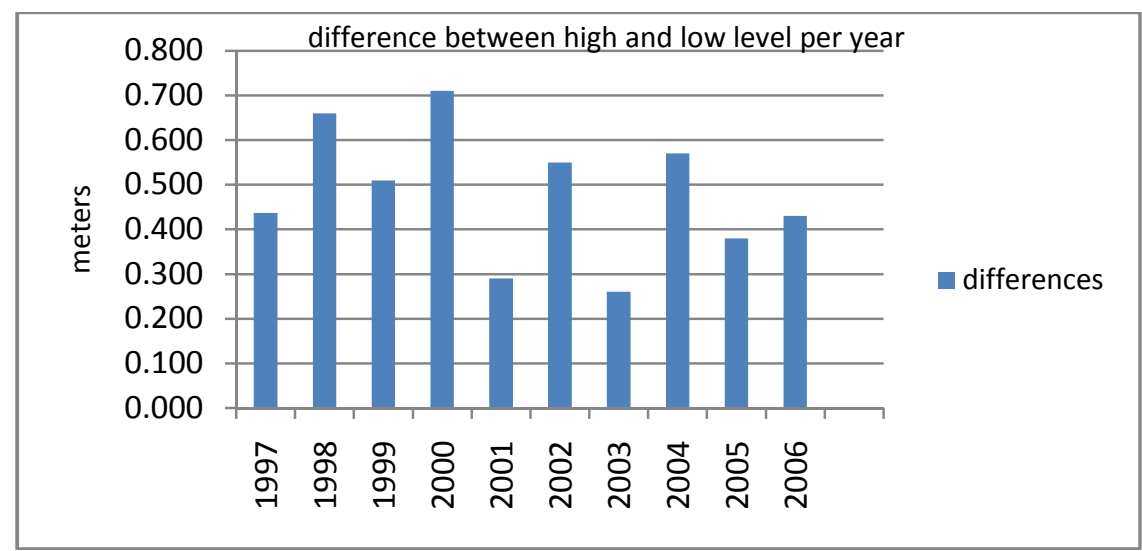

Figure 2: Differences between high and low level per year since 1997

The greatest difference between lowest and highest altitude is 0.710 meter measured in year 2000, this which means that a sensor with the range of 1 meter can be used to monitor this variation (J.C. Hamelain, 2005).

\section{System hardware architecture}

A Data Acquisition System is made of many components that are integrated to gather to sense a physical variable, by using transducers to convert the physical variable into an electrical signal and make it readable by an analog to digital converter(Perry Miller, 1999).

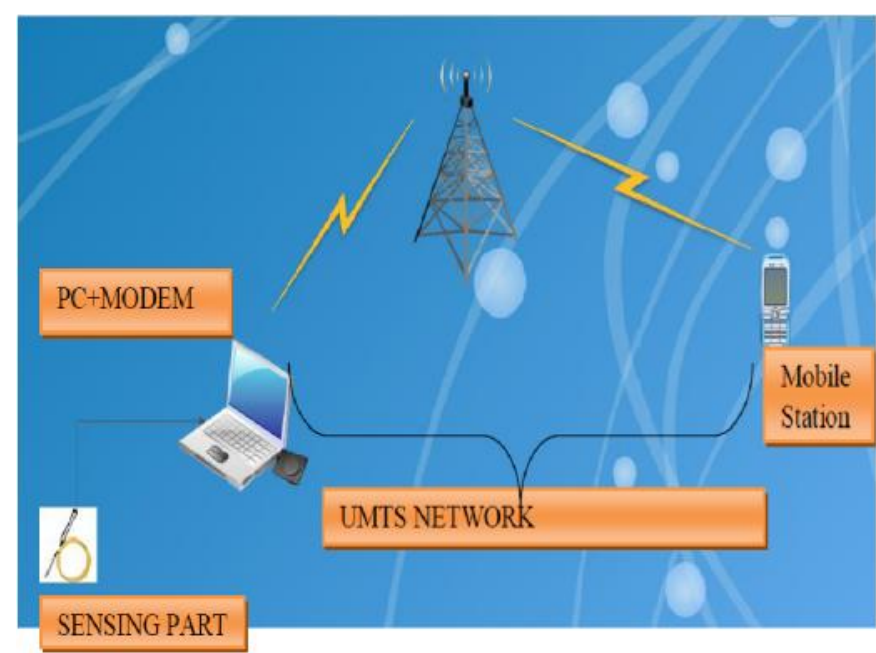

Figure 3: DAS network architecture 
The figure above shows the architecture of a Data Acquisition System. It is easy to identify the main parts: a mobile station, a UMTS network and a sensing part connected to a computer with a UMTS modem.

The figure 4 shows how data are taken from the physical parameter (the level of the Lake) to the final user, through signal conditioner, the Analog/digital converter and the computer which interprets the digital signal and give the needed information needed to the user(Edgar P. Casale, 2000).

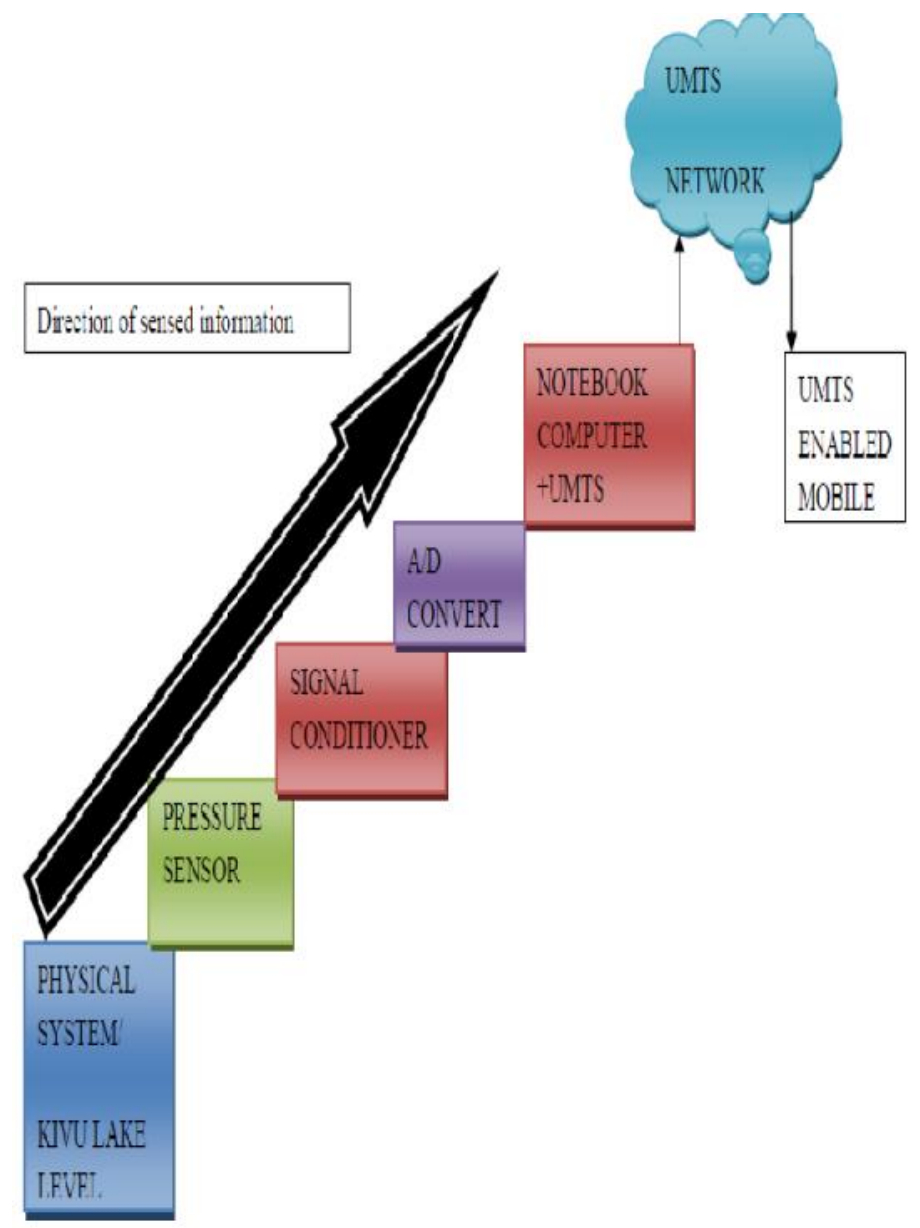

Figure 4: Sensing part details

In the network we designed, a pressure sensor acts as a transducer; it generates a signal as a function of the pressure imposed. For the purposes of this work, such a signal is electrical. 
The figure 5 represents a Submersible Pressure Transducer sensor for level and pressure(John Park and Steve Mackay, 2003) designed to be immersed in water, in order to translate variable pressure due to the change of the level of water into electric signal and then turned into digital signal to be interpreted by computer(Albert O.

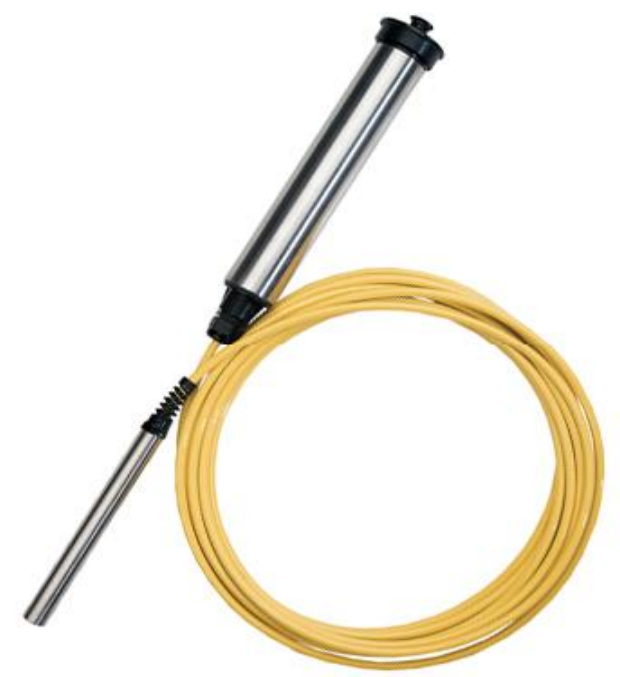

Grady, 2000).

Figure 5: WL400 Water Level Data Loggers.
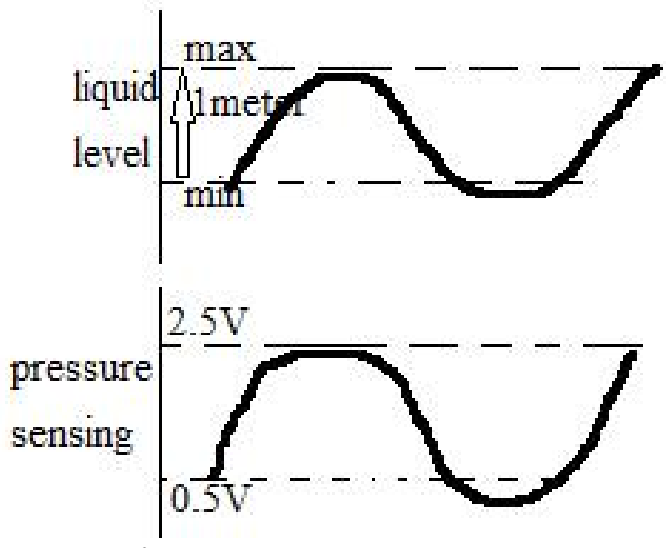

Figure 6: Proportion between level change and pressure sensed

A special A/D converter, a transducer and an amplifier, all are combined in this WL400 Water Level Data Loggers.

After a 1 meter range transducer has turned the instantaneous variable pressure into an analog electrical signal, and then the signal is amplified and turned into pulses. The WL400 Water Level Data Loggers is connected to one USB port of a computer and this signal will be used by the program to give the necessary information about the level of the lake.

The typical sensor considered in this paper is an all in one built, as you can see on the figure 5. The small end side is the one that contain the transducer under mechanical protection against physical disturbance. The used cable to 
connect the two end- sides is a two-wire $4-20 \mathrm{~mA}$ output signals that are linear with water depth. The transducer immersed in water is connected to the other part which must be placed outside water, seeming to be bigger on the other end. In this part all the other parts of the sensing part which are signal conditioner, $\mathrm{A} / \mathrm{D}$ converter are all combined and connected.

Therefore from this last side part, a USB cable adapted to it, is connecting the whole sensing part to the USB port of the computer. This is the digital signal entered into the computer to be interpreted by the program.

\section{Software Development and Simulation}

An operator sends a SMs to the number contained in the modem connected to a computer, then the Java SMSLib receives the SMs and immediately connect to a Java Application developed which in its turn check the input from the port connected to the sensor immersed in water. The input signal from sensor is analyzed and the corresponding level is captured and sent to the operator via SMSlib main class. Figure 7 and figure 8 show the structure of program used to send the information from the sensor to a computer.

The SMSLIB software we used in this work is built in two parts which are the main class and the simple class. The main class has the duty of receiving the request and then connects to the simple class to get information from the sensor and send it back to the destination address where information request was coming from.

The simple class is build in such way that a switch statement is inserted in order to be able to investigate and differentiate different input data from the sensor. For example if a 2 Volts signal is received from the signal, let the information to be sent to the operator be that the level is at 1469 meters of altitude, and once the Voltage is 3 Volts, let the information be that the level is at 1470 meters of altitude and so on.

The figure 7 shows the way taken by the signal requesting information from the operator and the information way back to operator. The figure 8 shows the flow chart of the program which is the sequence of the action or algorithm in other words.

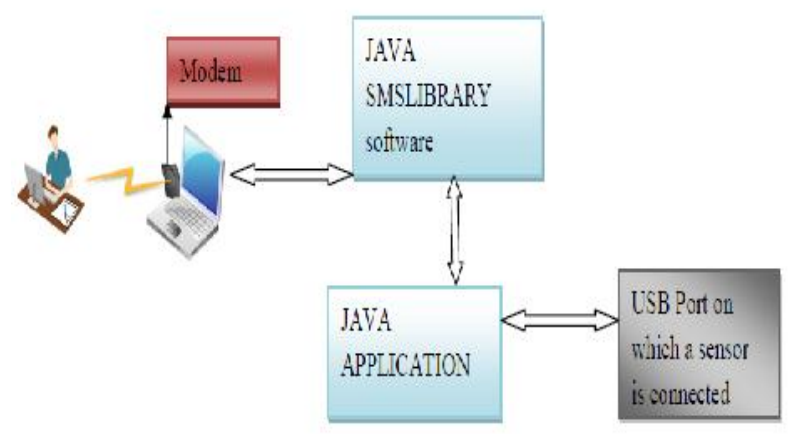

Figure 7: Software structure based on java SMlib 


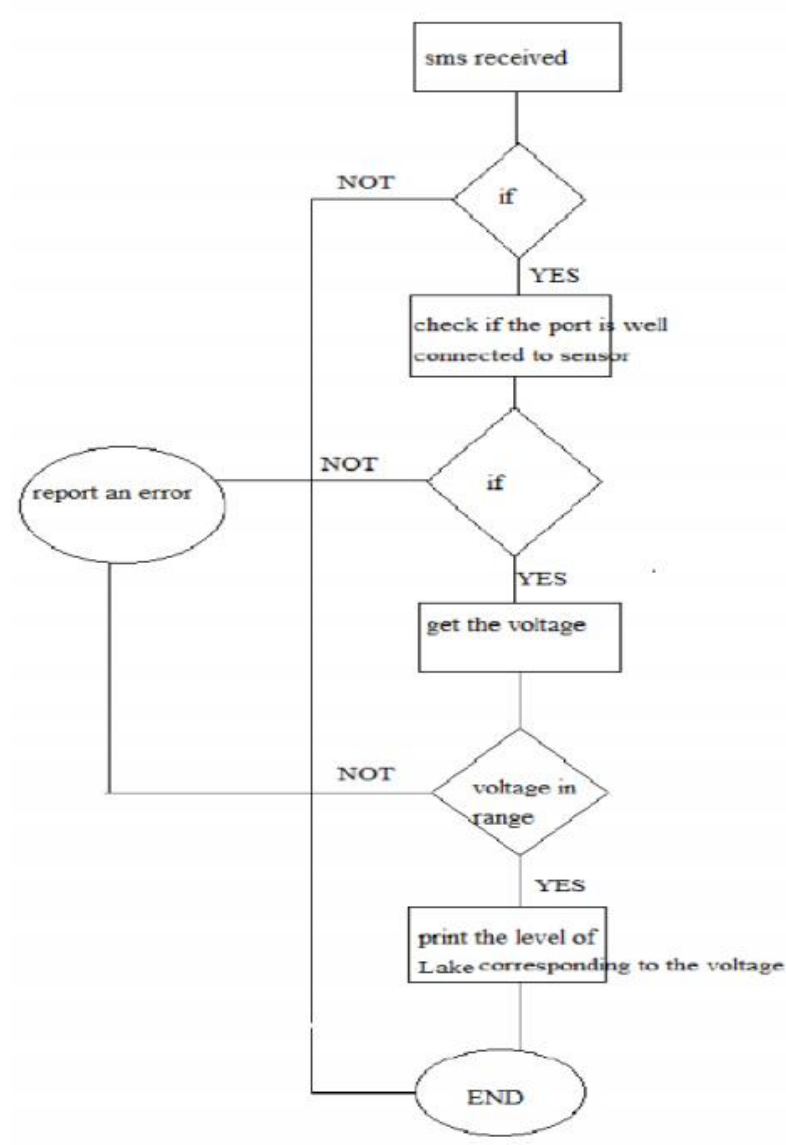

Figure 8: Flow chart of the program

In this flow chart, if an empty SMS is received on a computer from a mobile phone, the next step will be to check for the sensor connectivity to the other port of the computer, if no SMS received no action (Africa Information Technology Initiative, 2009).

Once the port is found to be well connected, then the input voltage is read, if voltage is not in programmed range, an error is reported. If the read voltage is in range, the corresponding level is printed and sent to the mobile phone.

For simulation applied software are the NetBeans software used as a software development platform and the SMSLib software, which detail about its usefulness is given in the next paragraph. Both softwares are installed on a PC working with windows 7 operating system. 
To get started with simulation first we create a new project under NetBeans on the following name org.aiti.sms. Under this project we create a package and a class, also on the same name as the project each. The next step will then be the connection to the sms library under SMSLib software installed and this gives us a two class java program; the main class and a simple class. Something very important here is that inside the simple is where we inserted a new program we designed to enable the simulation of our particular case study. The inserted program we developed is a switch statement which looks as follows (figure 9).

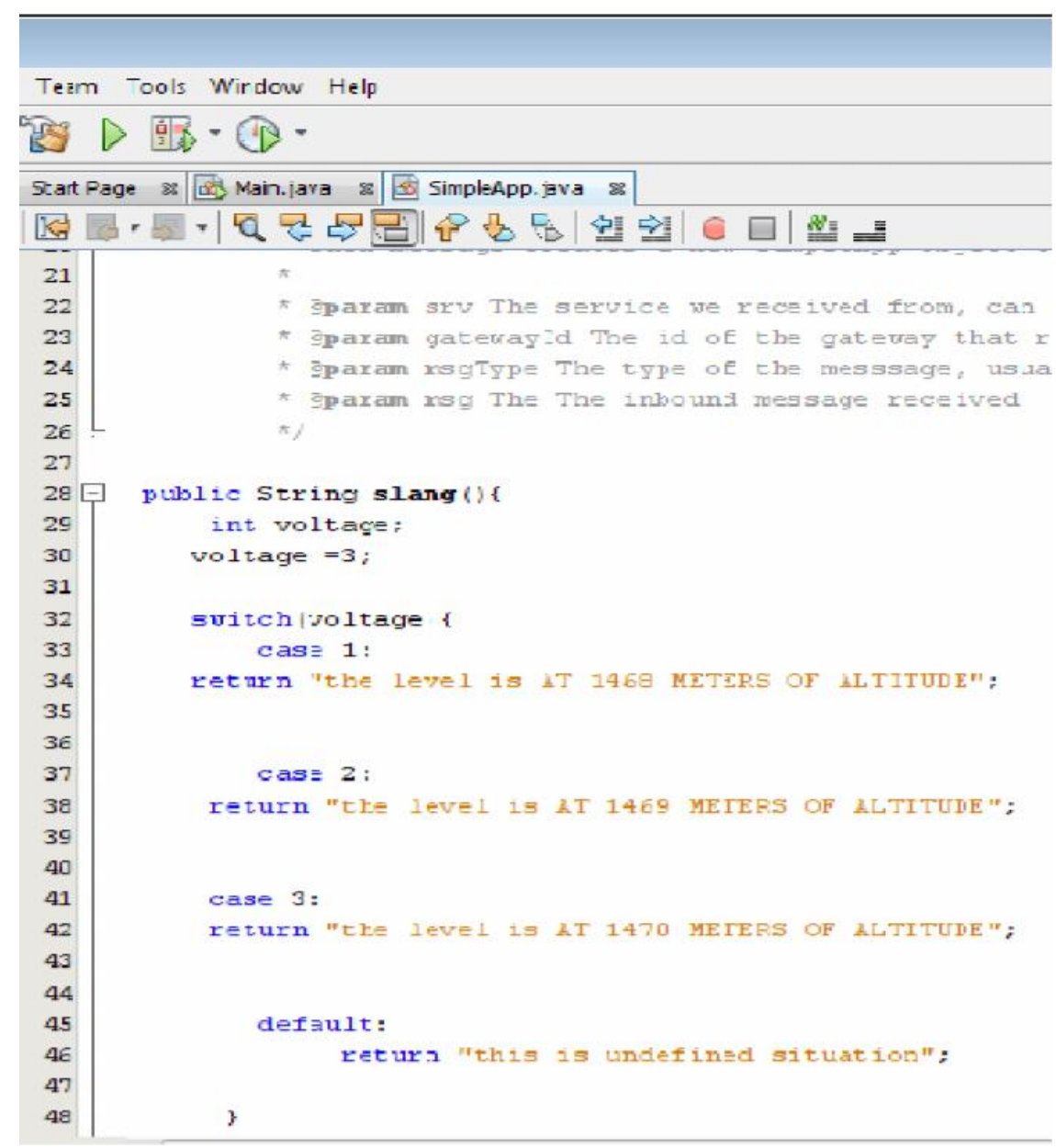

Figure 9: switch statement program

The output on a mobile phone will be looking like in figure 10 where the level of lake is converted in altitude in meter. 


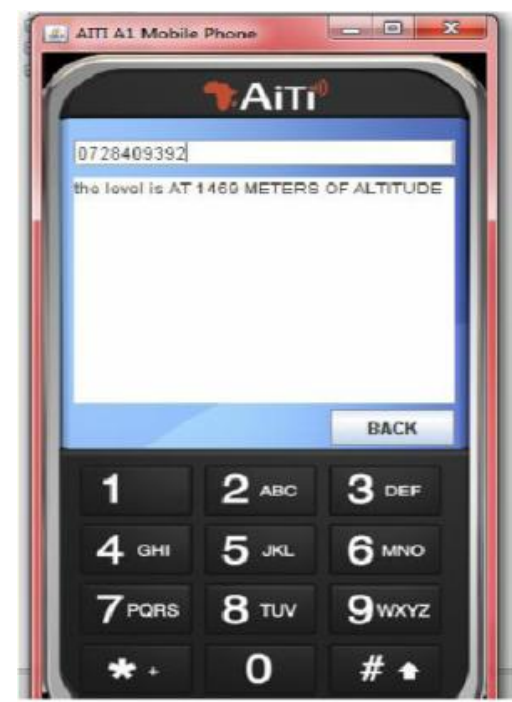

Figure 10: displayed level on a mobile phone

\section{Conclusion}

The main problem that triggered the thought about this conception was the daily need of the KIVU lake level measurement, this in order to optimize the use of resource while keeping natural environmental resource safety during the production of electricity by SINELAC/CEPGL. The major focus of this paper was to design a remote lake-water-level measurement data acquisition system that is Sensor, SMSlib software and UMTS network based.

A tree layer system is designed to achieve this work: a mobile station layer, UMTS network layer and a sensing part layer. The system will be more flexible than using server based network. The system accomplish the function of data processing and transmitting by the use of SMSlib software and a java application developed to run within this open source software on a PC.

From a mobile phone, via a UMTS network layer an sms is sent to a computer (PC) on which a pressure sensor immersed in water and connected on other terminal, then the computer replies to the request using an SMs after processing information about the level of water received from the sensor. The partial simulation of this system provides a proof of a successful way toward a full solution required.

Being a low cost, good and high performance and easy maintainable data acquisition system based on SMSlib software, this type of data acquisition system can be applied not only in the fields of hydrological processes, but 
also in fields like civil engineering and in some more industrial requirements solutions.

For the future, we want to implement this technology for SINELAK/CEPGL for controlling remotely the Kivu lake level. This can help to know exactly the annual variation of the level of this lake and make good plannification for the power plants.

\section{References}

1. Microsoft(2009), Student with Encarta premium.

2. Perry Miller(1999), Aspects of Data Acquisition Systems Design.

3. Txomin Nieva \& Alain Wegman(2000), A conceptual Model for Remote Data Acquisition systems, proceeding of the $19^{\text {th }}$ international conference on conceptual modeling, Salt Lake City, Utah, USA.

4. Edgar P. Casale(1996), Data acquisition system fundamentals, The Ohio state university, College of Engineering, Engineering H192, Hands-on Lab

5. Wiley and Maruzen(2002), WCDMA Mobile Communications systems.

6. Naral Dalal(2001), A comparative study of UMTS (WCDMA) and CDMA2000 networks.

7. John Park and Steve Mackay(2003), Practical Data Acquisition for Instrumentation and control Systems.

8. J.C Hamelain(2005), Liquid Level Control Using a Pressure Sensor.

9. Global Water(2009), WL 400 water level Sensor, submersible pressure transducer for monitoring level and pressure, Instrumentation resource book, www.globalw.com,11320 Amalgam way Gold river ,CA 95670

10. CCNA Discovery Learning Guide(2011), Designing and Supporting Computer Networks.

11. Albert O'Grady(2000), Transducer Sensor Excitation and Measurement Techniques.

12. Africa Information Technology Initiative(2009), MIT AITI Mobile Development in Java SMS Hardware Set-Up Information. 